\title{
La philosophie en Espagne, en « crise perpétuelle » (1850-1940)?
}

\section{Camille Lacau St Guily}

\section{(2) OpenEdition}

1 Journals

\section{Édition électronique}

URL : https://journals.openedition.org/cher/3495

DOI : $10.4000 /$ cher.3495

ISSN : 2803-5992

\section{Éditeur}

Presses universitaires de Strasbourg

\section{Édition imprimée}

Date de publication : 1 décembre 2015

Pagination : 53-62

ISBN : 978-2-86820-913-9

ISSN : 1968-035X

\section{Référence électronique}

Camille Lacau St Guily, «La philosophie en Espagne, en « crise perpétuelle » (1850-1940) ?»,

reCHERches [En ligne], 15 | 2015, mis en ligne le 01 décembre 2021, consulté le 15 décembre 2021.

URL : http://journals.openedition.org/cher/3495; DOI : https://doi.org/10.4000/cher.3495

\section{(c) (i) (9)}

Ce(tte) œuvre est mise à disposition selon les termes de la Licence Creative Commons Attribution Pas d'Utilisation Commerciale - Partage dans les Mêmes Conditions 4.0 International. 


\title{
La philosophie en Espagne, en "crise perpétuelle » (1850-1940)?
}

\author{
Camille Lacau St Guily \\ Université Paris-Sorbonne (CRIMIC)
}

\begin{abstract}
Hay una manera española de religión, hay un misticismo español, pintura española, teatro español, etc., pero no hay en este sentido filosofía española. (Alas 1896)
\end{abstract}

Le constat que fait Leopoldo Alas Clarín (1852-1901), dans La Publicidad, en 1896, est pour le moins désolant. L'(in)existence de la philosophie et de la figure du philosophe dans la cité espagnole est une problématique constante, dans l'histoire des idées en Espagne.

Incompétence à systématiser entraînant un «déficit doctrinal» (Serge Salaün)? Incapacité à traduire son aspiration contemplative en une élaboration abstraite? Tendance « aphilosophique», pragmatiste ou actionniste de l'Espagne? Manuel García Morente (1886-1942), grand spécialiste espagnol de la philosophie européenne, allemande et française particulièrement, dans une conférence qu'il donne, le 21 octobre 1934, au "Club espagnol» de Buenos Aires, sur le thème «La filosofía en España», s’interroge en ces termes:

Un país, una nación que, como la nuestra, ha dado de sí genios enormes, formidables, en la literatura, en la poesía, en la prosa, en el drama, en la historia, en la política, en la guerra, en las instituciones, en la pintura, en la escultura, ¿cómo es posible que no haya producido grandes filósofos? (García Morente I, 2, 1996: 413)

L'Espagne souffrirait presque d'une "virginidad filosófica» (Zambrano 1984: 220) et la philosophie espagnole serait dans une "crise perpétuelle», le philosophe apparaissant comme une figure mineure dans la «république des lettres» espagnole. Pourquoi donc si peu de philosophes espagnols ont-ils exercé un magistère intellectuel, non seulement dans leur pays, mais également à l'étranger? Ne chercherait-on pas en vain des paradigmes philosophiques

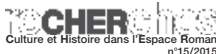


espagnols? Comment expliquer cette tendance à l'absence philosophique - crise perpétuelle - ou l'enlisement de ce pays, dans une crise structurelle, sur la large période qui sétend notamment de 1850 à 1940 environ? Pourquoi les Espagnols ont-ils toujours été plus ou moins désynchronisés des formes de la modernité philosophique et ont-ils pu s'attacher à des auteurs étrangers, parfois mineurs, qui n’ont pas exercé, hormis dans la Péninsule ibérique, le moindre ministère?

S'il semble que la philosophie se trouve en Espagne dans une constante situation critique, cette crise se révèle de plus en plus aiguë à la fin du XIX ${ }^{e}$ siècle et au début du $\mathrm{XX}^{\mathrm{e}}$. Cet accès dépressif, particulièrement violent à cette période, semble commencer à se dénouer et à se résorber, en 1910, au moment de la nomination de José Ortega y Gasset (1883-1955), à la chaire de métaphysique de l'Université Centrale de Madrid, puis plus largement dans les années 1910-1930. Même si Ortega y Gasset tente d'institutionnaliser la philosophie en Espagne, à travers la constitution d'une "école de métaphysique», l'«École de Madrid», on peut se demander s'il est réellement parvenu à mettre en place les conditions de l'émergence d'une philosophie «philosophique» (Ortega y Gasset 1956: 107) espagnole et donc à résorber cette crise. En réalité, il cherche à le faire moins comme philosophe systématique ou métaphysicien que comme journaliste, constatant ainsi que les Espagnols ne peuvent recevoir (il le dit lui-même) de leçons trop abstraites. Or, paradoxalement, il se tourne vers le paradigme le plus intellectualiste et conceptualiste de la philosophie européenne: la philosophie idéaliste allemande, envisagée comme régénérante pour le pays. Ce mariage qu’il tente de célébrer avec les Espagnols peut-il être fécond?

Quoi qu'il en soit, à peine quelques années plus tard, ces efforts d'institutionnalisation de la philosophie en Espagne sont anéantis. La guerre civile casse, en effet, cet élan nouveau et conduit au démantèlement non seulement de l' "École de Madrid», mais également du réseau d'intellectuels et de «philosophes» qui était apparu dans la constellation ortéguienne. La philosophie replonge dans une longue crise structurelle, tout comme la "philosophie espagnole» qui avait à peine eu le temps de commencer à poindre, dans les années 1910.

Toutefois, considérer que la philosophie est en crise structurelle dans le pays, n'est-ce pas être prisonnier d'un intellectualisme radical qui consiste à limiter la définition de la philosophie à un système de pensée, abstrait, et à l'opposer à toute forme de production artisanale poétique? La philosophie existe en Espagne, même dans les années 1850-1940, mais on ne la cherche sans doute pas là où elle se trouve, et Ortega est peut-être, pour une part, responsable d'avoir détourné l'attention historiographique de l'une des grandes trajectoires quaurait suivie ce que l'on pourrait appeler une "philosophie espagnole», notamment une philosophie poétique, vraiment réconciliatrice de la raison et de la vie, de la philosophie et de la poésie. 


\section{Une absence de prédisposition à la philosophie en Espagne au tournant du XIXe siècle?}

Comme le constate Manuel García Morente, dans le quotidien El Sol du 8 mars 1936 dans un article intitulé "Carta a un amigo: evolución filosófica de Ortega y Gasset », il n’existe pas, dans les années 1900, de philosophie proprement dite dans ce pays:

Por entonces, la filosofía en España no existía. Epígonos mediocres de la escolástica, residuos informes del positivismo, místicas tinieblas del krausismo, habían desviado el pensamiento español de la trayectoria viva del pensamiento universal, recluyéndolo en rincones excéntricos, inactuales, extemporáneos. España permanecía por decirlo así al margen del movimiento filosófico. $\mathrm{Ni}$ siquiera como simple espectador participaba en él. (García Morente I, 2, 1996: 539)

Comment expliquer l'inexistence ou l'inconsistance de la philosophie en Espagne, notamment dans les années 1850-1910, du moins son "extemporanéité» ou «inactualité » structurelles?

Tout au long du XIX ${ }^{e}$ siècle, particulièrement pendant la seconde moitié, l'intelligentsia espagnole se divise en deux camps opposés et crée, par cette bicéphalisation idéologique, ce que l'on pourrait considérer comme une impossibilité philosophique espagnole. En effet, dans le camp des "Anciens" ou conservateurs, la métaphysique espagnole est scolastique. Un phénomène de «néothomisation» s'accentue en 1879, avec la publication de l'Encyclique Aeterni Patris par le Pape Léon XIII (1810-1903). Ce dernier y restaure le magistère intellectuel de saint Thomas d'Aquin sur l'Église romaine. Les autres philosophies sont alors facilement considérées par les intellectuels catholiques espagnols comme une transgression sacrilège de cette nouvelle orthodoxie doctrinale.

Dans le camp europhile des «Modernes» - «front» qui aurait pu accueillir ou faire germer la philosophie moderne dans le pays -, le positivisme antimétaphysique et anti-spéculatif séduit de plus en plus les jeunes intellectuels. Il s'impose chaque fois plus clairement, précisément à partir de la fin des années 1870, comme une réponse "politique » et efficace à la métaphysique néothomiste d'un certain nombre d'intellectuels catholiques romains.

Par ailleurs, ces réformateurs qui ont participé pour beaucoup à la création, en 1876, de la Institución Libre de Enseñanza (ILE), au-delà de leur attrait pour un positivisme potentiellement régénérant ou «revertébrant» pour l'Espagne, ne semblent pas beaucoup apprécier la métaphysique spéculative. Manuel García Morente souligne également l'absence, dans les années 1900, chez nombre d'entre eux, de prédisposition pour la métaphysique, voire un sentiment de mépris:

Hacia $1900[\ldots]$ el positivismo reinante perseguía con implacable saña todo auténtico esfuerzo para pensar auténticamente; y había logrado sepultar bajo el ridículo y el menosprecio los más venerables vocablos de la vieja y eterna filosofía. La metafísica era considerada como ocupación de soñadores ociosos, una manera no siempre inocente de perder concienzudamente el tiempo. (García Morente 1972: 145) 
Ces «Modernes», de plus en plus séduits par le positivisme, sont sensibles à des pensées moins abstraites, plus immédiates et concrètes que ne l'est la métaphysique. Ils se tournent, pour beaucoup, vers des pensées "utilisables» en termes d'actions réformatrices. La pensée doit servir. Un certain nombre de ces intellectuels progressistes, en effet, sont dans une recherche pragmatiste. À ce propos, García Morente souligne, dans sa conférence sur "La filosofía en España», la particularité de la pensée espagnole, évoquant, d'une part, son manque d'appétence pour la systématisation philosophique, mais l'expliquant, d'autre part, par son pragmatisme intrinsèque, sa soif «vertébrante» d’action, d'enracinement dans le vivant par son désir d'élaboration et de fondation institutionnelles notamment:

El alma española pone por encima de la contemplación teórica especulativa intelectual la acción; pone por encima de la teoría la vida. [...]. El español ha preferido siempre vivir a pensar, o mejor dicho, el español ha puesto el pensamiento al servicio de la vida, lejos de poner la vida al servicio del pensamiento. [...]. Cuando el español siente une idea nueva, lo menos que se le ocurre es encastillarse, meterse dentro de sí mismo y darle una forma sistemática en un tomo de quinientas páginas, sino que lo que se le ocurre inmediatamente es fundar algo, fundar una orden religiosa, como Ignacio de Loyola, o fundar muchos conventos, como Santa Teresa. Los españoles intelectuales han tenido siempre almas de fundadores, porque de algún modo habían de convertir en vida esa idea. No pueden llevarla dentro de su pecho como mera idea. Mera idea es para el alma española algo inferior a la idea vivida, a la idea convertida en carne, hueso, vida, realidad. [...]. Por eso, en una época como la moderna, en donde la esencia del pensar filosófico consistía precisamente en lo contrario, en reducir toda realidad y toda la vida al pensamiento, el alma española no podía participar en esa manera de enfocar el problema filosófico. [...] el modo de ser propio del español es anteponer la acción y la vida a la meditación. (García Morente I, 2, 1996: 414)

À peine quelques années plus tard, la philosophe María Zambrano (19041991), dans son livre Pensamiento y poesía en la vida española de 1939, tente de définir la spécificité de la pensée espagnole. Pour elle, elle est un «réalisme». Elle l'appelle également "matérialisme». Désintéressés par la systématisation abstraite et l'élaboration conceptuelle, les Espagnols cultiveraient une fascination pour la réalité extérieure, la matière qu'ils se contenteraient de suggérer ou "évoquer» et non pas d’analyser. Ils seraient fondamentalement anti-théoriques et les inspirateurs d'une pensée "autre», originale. Ainsi, après avoir défendu l'idée que «el entendimiento español» est «inepto para la filosofía sistemática», elle développe une thèse très semblable à celle de García Morente:

El realismo español será ante todo un estilo de ver la vida y en consecuencia de vivirla, una manera de estar plantado en la existencia. No hay nada, ningún dogma de este "realismo" que nos permita cómodamente situarlo, enfrentarnos con él y analizarlo. No, nunca las cosas españolas son tan cómodas. El realismo, nuestro realismo insobornable, piedra de toque de toda autenticidad española, no se condensa en ninguna fórmula, no es una teoría. Al revés; lo hemos visto surgir como "lo otro" que lo llamado teoría, como lo diferente e irreductible a 
sistema. Intentar sistematizarlo sería hacerle traición, sería suplantarlo por una fría, muerta máscara; sería traer en vez de la viva substancia, su hueco molde. No hay fórmula, no hay sistema que compendie el realismo, nuestro arisco e indómito realismo y nos permita traerlo como un cadáver a la sala de disección del pensamiento; nos hemos de contentar si es que la fortuna nos ayuda, con evocarlo $^{1}$. (Zambrano 1939: 32-33)

Or, l'un des grands symptômes de cette absence d'appétence pour la philosophie, du «réalisme» et du «pragmatisme» dominants dont font le constat entre autres García Morente et Zambrano, se révélerait paradoxalement dans le fait de sêtre tourné, au XIX ${ }^{e}$ siècle, vers l'idéalisme krausiste et de l'avoir érigé en paradigme philosophique de la modernité intellectuelle espagnole. En concédant tant d'importance, dès la seconde moitié du XIX ${ }^{\mathrm{e}}$ siècle, à une philosophie a priori mineure, obsolète, épigonale du kantisme, et pas même reconnue en Allemagne, son pays de naissance, les intellectuels espagnols montrent, d'une certaine façon, leur manque d'intérêt pour la philosophie vivante².

D’ailleurs, la philosophie moderne, appelée "philosophie nouvelle», actuelle à la fin du XIX ${ }^{\text {e }}$ siècle, dont Henri Bergson (1859-1941) constitue la figure de proue, n'enthousiasme pas initialement les Espagnols (Lacau St Guily 2015: 21-61). En témoignent le mépris, l'hostilité et l'isolement dont va souffrir Leopoldo Alas Clarín (1852-1901), l'une des rares figures espagnoles, avec Giner de los Ríos (1839-1915), Miguel de Unamuno (1864-1936), et quelques autres, à s'intéresser à ce courant de renouveau, à la fin du XIX ${ }^{\text {e }}$ siècle. En effet, en 1897, Alas présente à l'Athénée de Madrid - supposé vivier de l'intelligentsia avantgardiste espagnole -, une série de conférences sur ce thème. Laccueil quelle lui réserve est désastreux, ce qui confirme le peu d'intérêt pour la philosophie moderne, symptôme d'une forme de désamour structurel des Espagnols pour la philosophie, en somme une tendance aphilosophique. Par son incapacité à faire fructifier la métaphysique, Clarín considère son pays comme un «campo estéril» (Alas 1899).

Il faut attendre plusieurs années pour que ce rejet de la philosophie et la crise structurelle quelle traverse s'estompent en Espagne. C'est notamment la nomination de José Ortega y Gasset à la chaire de métaphysique de l'Université Centrale de Madrid, qui non seulement favorise l'introduction dans ce pays de

la philosophie européenne et mondiale³, mais permet également l'émergence

1 Zambrano dira aussi à propos de la métaphysique: "El español la ha hecho, pero más que en libros sistemáticos, en un modo disperso: en poesía, en novela, en refranes, en coplas y hasta en silencios." (Zambrano 1984: 207)

2 Si Zambrano reconnaît "las espléndidas consecuencias para la vida social y cultural española" du krausisme, elle ajoute toutefois: "Mas la intención específicamente filosófica quedó fallida." (Zambrano 1984: 220) Les intellectuels en Espagne qui se revendiquèrent krausistes n'ont, pour la plupart, pas lu les écrits du philosophe.

3 Comme le dit Zambrano, dans l'un des cours donné à la Havane «Ortega y Gasset y la Filosofía actual», et reproduit dans Andalucía. Sueño y realidad: «¿Por qué nos resulta tan extraño que un español se decidiera a hacer filosofía y no otra cosa en aquella 
d'une philosophie espagnole. Soulignons, cependant, comme le rappelle García Morente, dans sa conférence de 1934, que le Catalan Jaime Balmes (1810-1848), Sanz del Río (1814-1869) et les héritiers des Lumières européennes, comme Giner de los Ríos, spécialement à travers le courant de la Institución Libre de Enseñanza, ont été les premiers initiateurs de la philosophie contemporaine en Espagne, en s'inspirant de paradigmes intellectuels étrangers, particulièrement européens, dont ils se font les "passeurs» (García Morente 1996 I, 2: 413)4. Cependant, ce mouvement ne semble pas faire beaucoup d'émules et le germe philosophique ne paraît alors pas réellement prospérer sur l'aride terre espagnole.

\section{José Ortega y Gasset ou l'institutionnalisation d'une «école philosophique » espagnole?}

L'un des grands mérites de José Ortega y Gasset est d'avoir cherché à s'adapter aux "circonstances espagnoles», au manque de prédisposition à la pensée systématique et d'avoir tenté de parler aux Espagnols, non de manière intellectualiste mais journalistique. Cependant, malgré cette adaptation, Ortega n'en est pas moins ambitieux pour son pays et vise l'enracinement de ce que Julián Marías (1914-2005) - ancien élève d'Ortega - nomme la "philosophie rigoureuse », García Morente, la "philosophie authentique », et Ortega lui-même, la "philosophie philosophique». Ce dernier nourrit donc un rapport ambigu à l'intellectualisme philosophique qui le fascine, tout en sachant qu'il a tendance à rebuter les Espagnols.

Mais d'abord, Ortega souhaite se plier aux "circonstances» de son pays, faisant le constat de son incapacité a priori à philosopher "philosophiquement». Ainsi, dans le "Prólogo a una edición de sus obras", il insiste sur son souci pragmatique d'adaptation à cette spécificité espagnole:

Mi vocación era el pensamiento, el afán de claridad sobre las cosas. [...] Hacia ese señorío de la luz sobre sí mismo y su contorno quería yo movilizar a mis compatriotas. [...].

Pero esta propaganda de entusiasmo por la luz mental - el lumen naturale había que hacerla en España según su circunstancia impusiera. En nuestro país, ni la cátedra ni el libro tenían eficacia social. Nuestro pueblo no admite lo distanciado y solemne. Reina en él puramente lo cotidiano y vulgar. Las formas del aristocratismo "aparte" han sido siempre estériles en esta península. Quien quiera

fecha en que Ortega lo hizo a principios del siglo, fijemos 1902? ¿Acaso no había existido filosofía en España? Pues, ante ningún hecho análogo, acaecido en otro país europeo del rango de España, surgiría en nuestro anónimo tal extrañeza. Pues sí, ése era el caso: en España, no había filosofía.»(Zambrano 1984: 201)

4 García Morente ne manque pas de souligner l'importance d'Ortega y Gasset: «A mediados del siglo xix empezó a resurgir el pensamiento filosófico español. [...] Por de pronto podemos citar algunos nombres. Desde 1850 hasta hoy estos tres nombres: Balmes, don Julián Sanz del Río y don José Ortega y Gasset.» (García Morente 1996 I, 2: 413) 
crear algo - y toda creación es aristocrática - tiene que acertar a ser aristócrata en la plazuela. He aquí por qué, dócil a la circunstancia, he hecho que mi obra brote en la plazuela intelectual que es el periódico. (Ortega y Gasset 2010: 96; 98).

Ainsi, comme le souligne Julián Marías, dans Les Philosophes espagnols de notre temps, si Miguel de Unamuno a eu un rôle important pour inculquer le sens philosophique à certains intellectuels espagnols, «le mérite d'Ortega a été, à la génération suivante, de faire de ce goût de la philosophie une solide vocation philosophique et d'être à l'origine d'une véritable école philosophique qui fleurit aujourd'hui dans tous les pays de langue espagnole» (Cascalès 1957: 5). Ortega comprend que les Espagnols ne philosophent pas à la manière des Allemands: l'Espagne n'a pas eu de théoriciens tels que Kant ou Hegel. Selon Ortega, c'est de façon asystématique, avec l'aide du journal, de la conférence ou du «bavardage» dans les tertulias, qu'il faut apprendre aux étudiants à philosopher "philosophiquement», et désamorcer la crise philosophique dans laquelle se trouve le pays. Ortega déjoue donc, en fin stratège, la solennité de certains cadres académiques pour mener les jeunes à la "philosophie philosophique». Car Ortega ne veut pas que se développe chez eux une vocation à la philosophie "bavarde», poétique ou mystique, trois formes dérivées ou avatars de la philosophie, selon lui, auxquelles il est hostile.

Pour insuffler cet élan nouveau, il participe, en plus de son travail journalistique, à la mise en place du cadre d'une institution philosophique. En effet, dès 1910, avec sa nomination à la chaire de métaphysique de l'Université de Madrid, les fondations de l' «École de Madrid» sont posées ${ }^{5}$. La philosophie en Espagne progresse alors vers un âge d'or et ce, jusqu'en 1936. C’est une période riche qui souvre et pendant laquelle des esprits profonds, tels que José Gaos (1900-1969), Xavier Zubirí (1898-1983), María Zambrano (1904-1991), Julián Marías (1914-2005) et bien d'autres, sont formés aux «Lumières de la raison (vitale?)».

On peut, cependant, se demander si, pendant la période 1910-1936, Ortega parvient réellement à mettre fin à cette crise structurelle de la philosophie. En effet, pourquoi Ortega considère-t-il le journal ou la tertulia comme des moyens adéquats pour entrer en philosophie, tout en s'obstinant à inculquer

5 Comme le souligne José Luis Abellán, «la Escuela de Madrid no empezó a funcionar, con los caracteres de articulación institucional que señala Gaos, hasta la llegada de la Segunda República, dentro de la cual cabe acotar los años transcurridos entre 1933 y 1936 como los de establecimiento y máxima vigencia de la misma. Esto quiere decir que, en una primera perspectiva, cabe configurar como periodización inicial -aunque insuficiente- los años anteriores a 1933 -desde 1910, en que Ortega gana su cátedra de Metafísica en la Universidad de Madrid-como los antecedentes y los posteriores a 1936 - a consecuencia de la guerra civil- como los de disociación y exilio, de acuerdo con el siguiente esquema elemental:

1. Antecedentes (1910-1932).

2. Establecimiento (1933-1936).

3. Exilio y disociación (1936-1955).» (José Luis Abellán 1991: 11) 
aux Espagnols la philosophie la plus abstraite et rigoureuse qui soit, la philosophie de Kant notamment ${ }^{6}$, ce système qui prend parfois l'aspect d'une "prison» asphyxiante dont lui-même voudra s'extirper? La pensée idéaliste allemande pouvait-elle fondamentalement s'hispaniser? Pourquoi vouloir initier les Espagnols aux arides Lumières allemandes? Le rationalisme allemand, son «hyper-intellectualisme» pouvaient-ils structurellement et authentiquement s'implanter dans le cœur des Espagnols, si enclins à l'anti-intellectualisme, au vitalisme et/ou au mysticisme? Ortega, même s'il forgea le concept de «raison vitale» comme dépassement des excès du rationalisme et du vitalisme, fut-il un remède radical à cette crise? La question reste ouverte.

Certes, il convertit un certain nombre d'intellectuels à la philosophie systématique allemande, mais permit-il à la "philosophie systématique» de s'adapter à la terre espagnole et donc à une "philosophie espagnole» de se mettre en place? Il permit, en effet, à un certain nombre d'intellectuels d'avoir accès à une formation intellectuelle solide, mais favorisa peut-être par ailleurs, dans ce choix d’orientation philosophique, une production épigonale qui ne connut pas un rayonnement vraiment majeur, hors du magistère ortéguien.

\section{La Guerre civile ou le démantèlement de l'École de Madrid: la philosophie de nouveau en crise?}

C’est surtout la guerre civile qui éclate en 1936 et l'instauration de la dictature nationaliste, à partir de 1939, qui provoquent un brutal retour de la crise de la philosophie en Espagne. Elle commence par le démantèlement de l'École de Madrid, en tant qu'institution, et des réseaux d'intellectuels qu'elle avait permis de tisser. Dans l'exil intérieur ou extérieur, la philosophie espagnole se diffracte. À partir de cette date, elle ne peut germer qu'en dehors de l'Espagne. Toutefois, expatriée, la philosophie qui a éclos dans le pays, dès les années 1910, ne peut-elle pas perdurer? D’autant que la philosophie est-elle nationale?

De façon presque prémonitoire, García Morente dit à ce propos, dans la conférence qu'il donne à Buenos Aires, le 21 octobre 1934, que la philosophie est apatride:

La filosofía no tiene patria. Es un equívoco lamentable el de poner al sustantivo filosofía el adjetivo nacional. Equívoco lamentable, porque la filosofía es precisamente lo contrario del particularismo nacional, es universal (García Morente I, 2 1996: 412).

6 Comme le dit Zambrano, dans un article paru dans les Cuadernos de la Universidad del Aire à La Havane, en 1949, puis plus tard dans une biographie intitulée Unamuno, Ortega revient en Espagne avec une vocation philosophique qui prend source dans le néokantisme: «Pero lo que Ortega realizó al ir a estudiar denodadamente filosofía en la Escuela neokantiana de Marburgo, fue obra de amor filial que se dirige a la busca y la captura de aquello que más falta hace en el hogar paterno. No había filosofía en España cuando el muchacho José Ortega y Gasset siguió su vocación de españolfilósofo.» (María Zambrano 2003: 164) 
Les philosophes espagnols ont, en effet, pu continuer à se former, écrire et diffuser leur pensée dans des espaces publics autres qu'espagnols et la mêler à des flux philosophiques divers et universels.

Par ailleurs, un autre contre-argument remettrait en cause le caractère structurellement en crise de la philosophie espagnole. Lorsque l'on dit que la philosophie en Espagne est en crise perpétuelle, n'est-on pas prisonnier d'un intellectualisme radical qui consiste à dire que n'est philosophique que ce qui est systématique et abstrait? Si l'on accepte de considérer que la métaphysique peut être poétique et que la poésie peut être métaphysique, alors sans doute pourra$\mathrm{t}$-on affirmer que la philosophie n'est pas en crise en Espagne, et qu'il existe une philosophie espagnole vivante.

La «raison poétique» de María Zambrano, entre autres, n'est-elle pas un très bel exemple de la singularité de ce que peut être la philosophie espagnole, incarnée et non épigonale? La singularité de la pensée espagnole se révélerait, en effet, par d'autres biais que la philosophie stricto sensu. Mais l'Espagne ne se réclame pas de cette tradition spécifique et les hispanistes ne semblent pas très disposés à élargir leur conception de la philosophie. Or, certes, l'Espagne de la

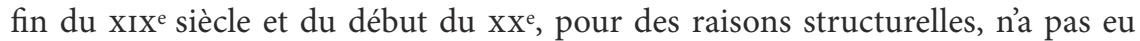
d'Husserl, de Bergson, d'Heidegger, et plus tard, pour des raisons également conjoncturelles, encore moins de Sartre, de Deleuze, de Foucault, de Derrida, de Lacan, parce que la pensée libre ne pouvait exister sous le franquisme.

Cependant, on ne semble pas avoir suffisamment conscience que la grande philosophie espagnole n'est pas strictement "philosophique». Ses philosophes ne sont peut-être pas ceux dont Ortega avait rêvé pour l'Espagne: des philosophes de système. Mais il faut avoir le courage de transgresser certains interdits, en déconstruisant notamment les fantasmes quavait nourris le Père Ortega pour sa progéniture. Sa disciple Zambrano paya très cher le fait d’avoir osé dépasser la Raison vitale ortéguienne. Elle pleura longuement cette rupture, mais accoucha d'une Raison musicale, vraiment vivante (Lacau St Guily 2013: 73-100). Elle défendit, contre son maître peut-être trop prisonnier de constructions verticales, l'idée que la "philosophie poétique» nétait pas inférieure à la philosophie "philosophique». Elle refusa dêtre l'esclave de tout intellectualisme et des fantasmes intellectuels ortéguiens nourris pour ce pays. Elle se battit toute son existence pour montrer que l'Espagne ne devait pas avoir honte de savoir parler de la vie, non comme une philosophe au sens orthodoxe du terme, mais comme une philosophe-poétesse.

L'Espagne est l'une des figures de proue en Europe de cette philosophie hétérodoxe, injustement méprisée. En nous libérant de ces préjugés intellectualistes et élitistes, nous ne trouverons plus la crise sur le chemin de l'histoire de la philosophie contemporaine dans ce pays, mais les fruits d'un grand Esprit musical proprement espagnol, une pensée de chair et de sang, qui n'a peut-être rien à envier à Nietzsche. 


\section{Bibliographie}

Abellán, J.L., 1991, La escuela de Madrid, un ensayo de filosofía, Madrid, Asamblea de Madrid.

Alas, L., 1896, La publicidad, 19/08/1896.

Alas, L., 1899, La publicidad, 01/02/1899.

Cascalès, C., 1957, L'humanisme d'Ortega y Gasset, Paris, PUF.

García Morente ,M., 1996, Obras Completas I (1906-1936), vol. 2, Madrid, ed. de Juan-Miguel Palacios, Rogelio Roviro, Fundación Caja Madrid, Anthropos.

García Morente, M., 1996, «La filosofía en España» [1934], Revista de filosofía, 15 , p. 3-16.

García Morente, M., 1936, El Sol, 08/03/1936.

García Morente, M., 1972, «Necrología», in: La filosofía de M. Bergson [1917], ed. Espasa Calpe, Madrid.

Lacau St Guily, C., 2015, Une histoire contrariée du bergsonisme en Espagne (1875-1930), Paris, L'Harmattan.

Lacau St Guily, C., 2013, María Zambrano, La tumba de Antígona y otros textos sobre el personaje trágico, Paris, PUF-CNED.

Marías, J., 1954, Philosophes espagnols de notre temps, Paris, Aubier.

Ortega y Gasset, J., 1956, El tema de nuestro tiempo [1923], Madrid, Revista de Occidente, $12^{\mathrm{e}} \mathrm{ed}$.

Ortega y Gasset, J., 2010, «Prólogo a una edición de sus obras» [1932], in: Obras completas, tomo V (1932-1940), Madrid, Taurus.

Zambrano, M., 1984, Andalucía. Sueño y realidad, Granada, Biblioteca de la cultura andaluza.

Zambrano, M., 1991, Pensamiento y poesía en la vida española [1939], México, El Colegio de México.

Zambrano, M., 2003, Unamuno, Barcelona, Debolsillo. 